\title{
Efectividad de 5-fluoruracilo y vinorelbina en pacientes multitratadas por cáncer de mama metastásico
}

\author{
José Luis González Vela, Jorge Martín Sánchez Guillén, Sergio Arnoldo Treviño Aguirre, David \\ Hernández Barajas, William Orlando Brito Villanueva, Eloy Cárdenas Estrada y Juan Francisco \\ González Guerrero \\ Centro Universitario contra el Cáncer, del Hospital Universitario. Universidad Autónoma de Nuevo León. México
}

\begin{abstract}
Objetivo. Evaluar la actividad y toxicidad de fluoruracilo en infusión continua y vinorelbina en segunda o tercera línea de tratamiento del cáncer de mama metastásico (CMM).

Método y pacientes. En este estudio fase II se incluyeron 24 pacientes que habían recibido doxorrubicina y/o paclitaxel. Se administró 5-fluoruracilo a $1 \mathrm{~g} / \mathrm{m}^{2} /$ día en infusión continua por 3 días y vinorelbina a $30 \mathrm{mg} / \mathrm{m}^{2}$ D1 cada 21 días por 6 ciclos.

Resultados. Las respuestas globales observadas fueron del 37,5\% (12,5\% respuestas completas). El período libre de enfermedad se calculó una media de 6,33 $\pm \mathbf{8 , 1 2}$ meses (IC 95\% de 3,43 meses). Se observó toxicidad en el 12,5\% de las pacientes y no se registró toxicidad grave ni muertes relacionadas a tratamiento.

Conclusión. EI 5-fluoruracilo/vinorelbina a las dosis administradas es un esquema efectivo en pacientes con CMM multitratadas, con un bajo perfil de toxicidad y costo.
\end{abstract}

Palabras clave: cáncer de mama, metástasis, 5-fluoruracilo, vinorelbina.

González Vela JL, Sánchez Guillén JM, Treviño Aguirre SA, Hernández Barajas D, Brito Villanueva WO, Cárdenas Estrada E, González Guerrero JF. Efectividad de 5-fluoruracilo y vinorelbina en pacientes multitratadas por cáncer de mama metastásico. Clin Transl Oncol. 2005;7(10):441-6.

\section{Efectiveness of 5-fluoruracil and vinorelbine in patients who had received multi-treatments for metastatic breast cancer}

Purpose. In this study we evaluated the activity and toxicity of a combination of 5-fluoruracil continuous

Correspondence: José Luis González Vela.

Centro Universitario contra el Cáncer, del Hospital Universitario.

Universidad Autónoma de Nuevo León.

Avenida Madero y Gonzalitos, s/n.

Colonia Mitras Centro.

Monterrey, Nuevo León, México.

E-mail: josegonzalezvela@hotmail.com

E-mail: jorgesanchezguillen@hotmail.com

Received 11 May 2004; Revised 19 April 2005; Accepted 20 May 2005. infusion and vinorelbine as second or third line chemotherapy in metastatic breast cancer (MBC).

Patients an methods. A total 24 patients who had received doxorrubicin and/or paclitaxel were included in this study. The regimen consisted in 5-fluoruracil $\operatorname{lgr} / \mathrm{m}^{2}$ BSA continous infusion for 3 days and vinorelbine $30 \mathrm{mg} / \mathrm{m}^{2}$ intravenous (IV) on day 1 . The cycles were repeated every 21 days for 6 cycles.

Results. Objective responses were recorded in $\mathbf{3 7 . 5} \%$ (12.5\% complete remission). The median deseasefree survival was calculated $6.33 \pm 8.12$ months (CI 95\% of 3.43 months). Toxicity was observed in 12.5\% of the patients and no treatment related deaths were recorded.

Conclusion. The combination of 5-fluoruracil/vinorelbine at the dose administered is an effective regime in patients with MBC, with low toxicity and cost.

Key words: Breast cancer, metastasis, 5 fluoruracil and vinorelbine.

\section{INTRODUCCIÓN}

En México la incidencia de cáncer de mama en el año 2000 fue de 9.998 nuevos casos con una tasa de 19,8/100.000 habitantes con 3.503 defunciones ${ }^{1}$. En los EE.UU. se estiman 5 casos por cada 100.000 en mujeres de 25 años, 150/100.000 a los 50 años y 200/100.000 a los 75 años de edad ${ }^{2,3}$. La quimioterapia desempeña un papel importante en el tratamiento del cáncer de mama metastásico al ofrecer la posibilidad de la paliación y prolongar la supervivencia ${ }^{4-7}$. En el tratamiento del cáncer de mama metastásico, las antraciclinas son fármacos muy activos además del paclitaxel, docetaxel, gemcitabina y vinorelbina; sin embargo cuando los pacientes no responden bien a estos medicamentos, tienen un pobre pronóstico y sólo un pequeño porcentaje alcanza respuestas objetivas con otras líneas de tratamiento ${ }^{8,9}$. Cada uno de ellos con perfiles diferentes de toxicidad, método de aplicación y coste, estos fármacos de segunda línea conducen a respuestas clínicas y períodos libres de progresión muy similares, aunque la vinorelbina tiene el menor coste por ciclo de tratamiento ${ }^{9,10}$. Esta última es un fármaco derivado 
de los alcaloides de la vinca, que utilizada en primera línea tiene un índice de respuestas globales de un 40\% a $60 \%{ }^{10-12}$. La toxicidad más frecuente e importante que limita su empleo es la neurológica y la hematológica $^{13-16}$.

El 5-fluoruracilo (5FU) tiene una menor toxicidad cuando se administra en infusión continua (IC) que en bolos. Actúa específicamente en la fase $\mathrm{S}$ del ciclo celular, con una vida media corta de 10 a 20 minutos, lo que hace que su actividad sólo ocurra en una pequeña fracción de células tumorales en esta fase. Cuando el fármaco es administrado durante un período prolongado de tiempo, un mayor número de células en división están expuestas al mismo, lo cual hace pensar en una mayor efectividad al administrarlo en $\mathrm{IC}^{17,18}$.

Varios estudios han demostrado que el 5FU administrado en IC a dosis $>450 \mathrm{mg} / \mathrm{m}^{2}$ superficie corporal (SC) conducen a índices de respuesta superiores al $53 \%$ en primera línea de tratamiento ${ }^{17,18}$. En segunda línea, los índices de respuesta son de un $\sim 30 \%{ }^{19-22}$. El $5 \mathrm{FU}$ cuando se administra en IC es menos mielotóxico, lo que permite combinarlo con otros fármacos como la vinorelbina.

La asociación de 5FU/vinorelbina tiene ventajas teóricas que son: mecanismos diferentes de citotoxicidad, resistencia y una baja sobreposición de sus efectos tó$x i \cos ^{17,18}$. En seis estudios fase II se ha reportado la efectividad y toxicidad de esta combinación, todos ellos con dosis diferentes de fluoruracilo y vinorelbina ${ }^{23-26}$. En nuestro estudio, con el fin de minimizar los efectos tóxicos pero sin restar efectividad, redujimos la frecuencia de vinorelbina a cada 21 días y se compensó con 5FU en infusión continua.

Los objetivos primarios de este estudio fueron evaluar la eficacia y toxicidad del 5FU/vinorelbina en segunda o tercera línea de tratamiento en pacientes con cáncer de mama metastásico con pobre respuesta a doxorrubicina y/o paclitaxel.

\section{METODOLOGÍA DEL ESTUDIO}

Estudio fase II observacional, no comparativo, prospectivo. Los pacientes fueron elegibles si tenían el diagnóstico histopatológico de adenocarcinoma de mama, clasificado como enfermedad metastásica, ya sea por recaída o por cualquier $\mathrm{T}$ o $\mathrm{N}$, pero M1 (de la American Joint Comittee on Cancer [AJCC]). Se incluyeron todos los pacientes que acudieron para atención médica, que reunían los criterios de inclusión y exclusión (descritos más abajo) en el período de septiembre de 1999 a junio de 2002, en el Centro Universitario contra el Cáncer. El estudio fue aprobado por el Comité de Ética del Hospital Universitario, de la Universidad Autónoma de Nuevo León, y se obtuvo el consentimiento informado en todos los casos.

Para llevar un seguimiento y evaluar el tiempo de supervivencia, se utilizó el procedimiento de producto li- mitado de Kaplan-Meier, sin datos censados (censurados), con tiempos de inicio diferentes según cada paciente. El rango de días desde la primera hasta la última consulta fue de 24 y 1.433 días. Se utilizó el paquete estadístico SPSS/PC (SPSS inc. Chicago IL) para el cálculo de evento, probabilidad de sobrevivencia acumulada $(\mathrm{S}(\mathrm{t}))$, error estándar de $\mathrm{S}(\mathrm{t})(\mathrm{SE}(\mathrm{S}(\mathrm{t})))$, así como el intervalo de confianza de 95\% (IC-95\%) y el riesgo $(\mathrm{H}(\mathrm{t}))$. Se calculó la mediana así como el IC$95 \%$ y los percentiles 25,50 y 75 .

El objetivo primario de este estudio fue evaluar la eficacia y toxicidad del 5FU/vinorelbina en segunda o tercera línea de tratamiento en pacientes con cáncer de mama metastásico con mala respuesta a doxorrubicina y/o paclitaxel.

\section{Pacientes y métodos}

Se incluyeron un total de 24 pacientes con informe histopatológico de adenocarcinoma de mama, con enfermedad metastásica, medible o evaluable por examen físico y/o estudio de imagen, con mala respuesta al tratamiento con antraciclinas y/o taxanos, en estado funcional mayor del 60\% (escala Karnofsky), las pacientes postmenopáusicas debían tener receptores hormonales de estrógeno y progesterona negativos o desconocidos, adecuada función renal (depuración de creatinina $\geq 50 \mathrm{ml} / \mathrm{min}$ ), hematológica (neutrofilos $\geq 1.500 / \mathrm{dl}$, plaquetas $\geq 125.000 / \mathrm{dl}$ ), hepática (bilirrubinas y transaminasas $\leq 1,5$ veces del valor superior normal) y expectativa de vida superior a 3 meses. Se excluyeron pacientes embarazadas y mentalmente incapacitadas.

\section{Evaluación pretratamiento}

Ésta incluyó historia clínica y examen físico completos, biometría hemática completa, perfil bioquímico, pruebas de funcionamiento hepático, el informe histopatológico de adenocarcinoma de mama y confirmación de enfermedad metastásica por biopsia y/o estudios de extensión, radiografía de tórax, ultrasonograma hepático, gamagrama óseo y en algunos pacientes tomografía axial computarizada.

\section{Quimioterapia}

La vinorelbina se administró por vía intravenosa (IV) en solución salina, a dosis de $30 \mathrm{mg} / \mathrm{m}^{2} \mathrm{SC}$ en un lapso de 15 minutos en el día 1 . El 5FU se administró en IC a $1 \mathrm{~g} / \mathrm{m}^{2}$ IV SC/día durante 3 días cada 21 días, con un mínimo de 1 y máximo de 6 ciclos. A todas las pacientes se les colocó catéter venoso central subclavio, de la marca Arrow (No CS24701E14GA) con la técnica de Seldinger (para la aplicación de la quimioterapia). La medicación prequimioterapia para prevenir la 
náusea y vómito consistió en ondansetrón o tropisetrón más dexametasona $8 \mathrm{mg}$ IV.

\section{Criterios de respuesta}

Las respuestas a tratamiento fueron evaluadas de acuerdo con los criterios de la Organización Mundial de la Salud (OMS), clasificadas en: a) respuesta completa (RC): ausencia clínica de toda enfermedad por cáncer después de tratamiento; b) respuesta parcial (RP): una disminución $\geq 50 \%$ de la suma del producto de los diámetros de las lesiones medibles después de tratamiento y sin aparición de nuevas lesiones $\geq 4$ semanas; y c) enfermedad estable (EE): es una disminución menor a una respuesta parcial y no reúne los criterios para progresión de la enfermedad, en un período de $\geq 4$ semanas.

\section{Criterios de progresión}

\section{Progresión de la enfermedad}

Incremento $\geq 25 \%$ en la suma del producto de los diámetros de las lesiones medibles o la presencia de nuevas lesiones o muerte del paciente como consecuencia del tumor.

Las evaluaciones fueron realizadas en cada ciclo de quimioterapia, por medio del interrogatorio clínico, examen físico y cada 3-4 ciclos con estudios de imagen. Todas las respuestas fueron evaluadas por personal médico de nuestro hospital (oncólogos, radiólogos, médicos especialistas en Medicina Nuclear). El seguimiento postratamiento se hizo de forma mensual hasta progresión de la enfermedad o muerte.

\section{Toxicidad al tratamiento}

La evaluación de la toxicidad al tratamiento fue hecha de acuerdo con los criterios de la OMS. La dosis de $5 \mathrm{FU} /$ vinorelbina se redujo un $25 \%$ cuando se presentó toxicidad grado III y IV.

\section{RESULTADOS}

En el período comprendido desde abril de 1999 a junio de 2002, fueron tratadas 24 mujeres con cáncer de mama metastático; la edad promedio fue de 54 años, con un rango de edad de 30 a 80 años, todas las pacientes tenían enfermedad metastásica y habían presentado fallo a una o dos líneas de tratamiento. El 67\% fueron pacientes posmenopáusicas; en este grupo de pacientes los receptores de estrógenos eran negativos o desconocidos.

Los sitios más frecuentes de metástasis fueron hueso (54\%), ganglios linfáticos (16\%), pleura y pulmones $(12,5 \%)$, hígado $(8,3 \%)$ (tabla 1$)$.

Se aplicaron un total de 91 ciclos de tratamiento; el promedio fue de 3,8 ciclos por paciente, con un rango
TABLA 1. Características de la población

\begin{tabular}{lc|c}
\hline Características & No de Pacientes & Porcentaje \\
\hline Ingresadas al estudio & 24 & \\
Edad en años & & \\
$\quad$ Media & 54 & \\
$\quad$ Rango & $30-80$ & \\
Estadio funcional (escala de Karnofsky) & \\
$90-100$ & 3 & $8 \%$ \\
$70-80$ & 14 & $58 \%$ \\
60 & 8 & $33 \%$ \\
Estado menopáusico & & \\
$\quad$ Premenopáusico & 8 & $33 \%$ \\
Posmenopáusico & 16 & $67 \%$ \\
Sitios de metástasis & & \\
$\quad$ Hueso & 13 & $54 \%$ \\
Tejidos blandos & 8 & $33 \%$ \\
Pulmones & 5 & $21 \%$ \\
Hígado & 3 & $12,5 \%$ \\
Encéfalo & 2 & $8 \%$ \\
No de órganos involucrados con metástasis & \\
1 & 14 & $58 \%$ \\
2 & 9 & $35,5 \%$ \\
$>3$ & 1 & $4 \%$ \\
\hline
\end{tabular}

de 1 a 6 ciclos, en tres pacientes se indicó como segunda, en 20 como tercera y en una como cuarta línea de tratamiento.

\section{Eficacia del tratamiento}

De las 24 pacientes tratadas el índice de respuesta se calculó una media de 38,33 \pm 31,09\% (IC 95\%: de $25,20 \%$ a 51,46\%). En 6 pacientes se observó respuesta parcial y en 3 de ellas respuesta completa.

De las 6 pacientes (25\%) con respuesta parcial, 4 tenían metástasis a ganglios supraclaviculares y se observó respuesta parcial después del primer ciclo de quimioterapia (QT). Las otras 2 mujeres tenían tumor que afectaba a casi toda la mama y se observó una respuesta parcial después del primer ciclo.

En 3 pacientes (12,5\%) se observó respuesta completa. Una de ellas tenía una lesión única metastásica al hígado, al cuarto ciclo de QT presentó RC de la lesión. Otra paciente que tenía metástasis a los ganglios del hueco supraclavicular (HSC) presentó RC en tejidos blandos después del cuarto ciclo de quimioterapia La tercera paciente con metástasis a los ganglios del HSC presentó RC después del quinto ciclo de quimioterapia (tabla 2).

Para el período libre de progresión se calculó una media de $6,33 \pm 8,12$ meses (IC del 95\%: de 2,90 a 9,76 meses).

\section{Toxicidad al tratamiento}

De las 24 pacientes tratadas, $11(45,5 \%)$ presentaron toxicidad relacionada con el tratamiento de acuerdo 
TABLA 2. Respuesta al tratamiento de acuerdo con sitio de metástasis

\begin{tabular}{l|r|c|c|cc}
\hline \multirow{2}{*}{ Sitio de metástasis } & \multirow{2}{*}{ EE } & \multirow{2}{*}{$\mathrm{RP}$} & \multirow{2}{*}{$\mathrm{RC}$} & \multicolumn{2}{|c}{ Total } \\
\cline { 5 - 6 } & & & & $\mathrm{n}$ & $\%$ \\
\hline Hueso & 9 & - & - & 9 & $37,5 \%$ \\
Hígado & 2 & - & 1 & 3 & $12,5 \%$ \\
Pulmonar/pleura & 3 & - & - & 3 & $12,5 \%$ \\
Ganglios HSC & - & 4 & 2 & 6 & $25 \%$ \\
Hueso y masa en la GM & - & 2 & - & 2 & $8,3 \%$ \\
HSC y pulmón & 1 & - & - & 1 & $4 \%$ \\
Total & 15 & 6 & 3 & & 24 \\
\hline
\end{tabular}

EE: enfermedad estable; RP: respuesta parcial; RC: respuesta completa; HSC: hueco supraclavicular; GM: glándula mamaria.

con los criterios de la OMS. En 5 pacientes hubo toxicidad grado I (20,5\%), toxicidad grado II en 3 pacientes (12\%), toxicidad grado III en 3 (12\%); no se observó toxicidad grado IV, ni muertes relacionadas con el tratamiento.

La toxicidad hematológica fue la más frecuente, se observó en 5 pacientes; una paciente presentó neutropenia no complicada grado III y otra grado I.

La toxicidad neurológica manifestada como neuropatía sensitiva se presentó en 2 pacientes, una de ellas fue grado II y la otra grado III; los síntomas se resolvieron al suspender el tratamiento.

La toxicidad gastrointestinal se presentó en 2 pacientes, una desarrolló mucositis grado II que mejoró al reducir el $25 \%$ de la dosis (de 5FU) y otra paciente tuvo náuseas, vómitos y diarrea grado II.

En 2 pacientes hubo dermopatía manifestada en una de ellas como lesiones vesiculopapulares dolorosas (además de mucositis descrita anteriormente) que mejoró con tratamiento local y reducción de la dosis de $25 \%$ del $5 \mathrm{FU}$ (tabla 3).

\section{DISCUSIÓN}

Aproximadamente del 6\% al 10\% de las pacientes con cáncer de mama se presentarán con enfermedad metastásica al momento del diagnóstico ${ }^{30}$. En este grupo de pacientes, la QT da un beneficio clínico a la mayoría de las enfermas, aunque esta enfermedad es esencialmente incurable. Los esquemas de QT son enfocados a la obtención de la mejor paliación de síntomas y con los mínimos efectos colaterales posibles. Aquellas pacientes con enfermedad metastásica que han presentado progresión a una primera y segunda línea de tratamiento, tienen un pronóstico menos favorable, con un índice de respuestas globales alrededor del 30\% $0^{21,23}$.

La combinación de 5FU/vinorelbina ha sido evaluada en cáncer de mama metastásico, en primera, segunda y tercera líneas, con índices de respuestas y grados de toxicidad variables (tabla 4); sin embargo estos resultados no pueden ser comparables, debido a diferencias en la dosis de tratamiento por ciclo y la población $\operatorname{tratada}^{16,17,19}$.

En nuestro estudio las pacientes fueron incluidas de manera prospectiva entre el período de septiembre de 1999 a junio de 2002, con cáncer de mama metastásico que no habían respondido a una o más líneas de tratamiento. El esquema empleado de $5 \mathrm{FU}$ a $1 \mathrm{~g} / \mathrm{m}^{2} \mathrm{SC}$ en IC durante 3 días y vinorelbina a $30 \mathrm{mg} / \mathrm{m}^{2} \mathrm{SC}$ cada 21 días, es novedoso a estas dosis; la decisión de reducir la dosis de vinorelbina fue tomada para disminuir la toxicidad del tratamiento, en un grupo de pacientes donde unos de los objetivos era la paliación. Los resultados obtenidos en nuestro estudio mostraron una actividad considerable, con un índice de respuestas globales del 37,5\%, $3(12,5 \%)$ respuestas completas y 6 (25\%) parciales, con una media de 38,33 \pm $31,09 \%$ (IC $95 \%$ de 13,13\%). El período libre de enfermedad se calculó como una media de 6,33 \pm 8,12 meses (IC 95\% de 3,43). Los resultados obtenidos son similares a los de otros estudios, en pacientes en tercera línea de tratamiento ${ }^{18,26}$, (tabla 4).

El esquema de tratamiento fue bien tolerado y los resultados mostraron una baja toxicidad; esto es así probablemente por el esquema adoptado al administrar 5FU en IC y la dosis de vinorelbina cada 21 días. Se observó toxicidad grado III en el 12,5\% de los pacientes, siendo la hematológica la más frecuente seguida por la neuropatía. Es importante resaltar esto en comparación con los efectos tóxicos publicados en otros estudios ${ }^{16,17,20}$ los índices de toxicidad grado III y IV en el $65 \%$ al $90 \%$ de los pacientes tratados en un grupo de

TABLA 3. Toxicidad por tratamiento de acuerdo con los criterios de la OMS

\begin{tabular}{|c|c|c|c|c|c|c|c|c|c|c|}
\hline \multirow{2}{*}{ Efecto tóxico } & \multicolumn{2}{|c|}{ Grado I } & \multicolumn{2}{|c|}{ Grado ॥ } & \multicolumn{2}{|c|}{ Grado III } & \multicolumn{2}{|c|}{ Grado IV } & \multicolumn{2}{|c|}{ Total } \\
\hline & $\mathrm{n}$ & $\%$ & $\mathrm{n}$ & $\%$ & $\mathrm{n}$ & $\%$ & $\mathrm{n}$ & $\%$ & $\mathrm{n}$ & $\%$ \\
\hline Anemia & 3 & 12,5 & - & & - & & - & - & 3 & $12,5 \%$ \\
\hline Neutropenia & 1 & 4 & - & & 1 & 4 & - & - & 1 & $8 \%$ \\
\hline Neuropatía & - & & 1 & 4 & 1 & 4 & - & - & 2 & $8 \%$ \\
\hline Mucositis & - & & 1 & 4 & - & & - & - & 1 & $4 \%$ \\
\hline Diarrea, vómito y náuseas & - & & 1 & 4 & - & & - & - & 1 & $4 \%$ \\
\hline Piel & 1 & 4 & - & & 1 & 4 & - & - & $\begin{array}{c}2 \\
11\end{array}$ & $\begin{array}{c}8 \% \\
45,5 \%\end{array}$ \\
\hline
\end{tabular}


TABLA 4. Resultados de los diferentes estudios publicados

\begin{tabular}{|c|c|c|c|c|c|c|c|}
\hline Autor & $\mathrm{n}$ & $\begin{array}{c}\text { Esquema } \\
\text { de tratamiento } \\
\left(\mathrm{mg} / \mathrm{m}^{2} \mathrm{sC}\right)\end{array}$ & $\begin{array}{l}\text { Línea de } \\
\text { tratamiento }\end{array}$ & $\begin{array}{l}R G \\
(\%)\end{array}$ & $\begin{array}{c}\text { PLP } \\
\text { (meses) }\end{array}$ & $\begin{array}{c}\text { SVM } \\
\text { (meses) }\end{array}$ & $\begin{array}{c}\text { Toxicidad } \\
(\%)\end{array}$ \\
\hline Dieras $^{23}$ & 63 & $\begin{array}{l}\text { 5FU } 750 \text { IC D1 a D5 } \\
\text { VNB } 30 \text { D1 y D5 }\end{array}$ & Primera & $65 \%$ & 8,4 & 23 & 90 \\
\hline Nole $^{24}$ & 45 & $\begin{array}{c}\text { 5FU } 350 \text { D1 a D3 } \\
\text { LV } 100 \text { D1 a D3 } \\
\text { VNB } 30 \text { D1 a D3 }\end{array}$ & Primera & $61 \%$ & 8 & - & 77 \\
\hline Stuart ${ }^{25}$ & 59 & $\begin{array}{c}\text { 5FU } 200 \text { IC D1 a D21 } \\
\text { VNB } 30 \text { D1 y D8 }\end{array}$ & $\begin{array}{l}\text { Segunda y } \\
\text { tercera }\end{array}$ & $54 \%$ & 4,5 & - & 65 \\
\hline Berrutti26 & 83 & $\begin{array}{c}\text { 5FU } 200 \text { IC D1 a D28 } \\
\text { VNB } 30 \text { D1 y D15 }\end{array}$ & $\begin{array}{l}\text { Segunda y } \\
\text { tercera }\end{array}$ & $61 \%$ & 15 & & 10 \\
\hline González et al & 24 & $\begin{array}{c}\text { 5FU } 1.000 \text { IC D1 a D3 } \\
\text { VNB 30 D1 }\end{array}$ & $\begin{array}{c}\text { Segunda } 12,5 \% \\
\text { y tercera } 83 \%\end{array}$ & $37,5 \%$ & 7,7 & & 12,5 \\
\hline
\end{tabular}

RG: respuestas globales; PLP: período libre de progresión; SVM: sobrevida media; SC: superficie corporal; IC: infusión continua.

pacientes donde uno de los objetivos es la paliación de la enfermedad.

El coste del tratamiento por cada ciclo fue de $\$ 500$ US aproximadamente, comparado con otros esquemas de tratamiento de menor coste, y comparados con otros esquemas de tratamiento donde se incluye al docetaxel y gemcitabina.

\section{CONCLUSIONES}

En el tratamiento del cáncer de mama metastásico no hay un esquema estándar en pacientes que han fallado en 2 líneas de tratamiento. Los resultados obtenidos de nuestro estudio mostraron una actividad moderada al obtener un índice de respuesta del 37,5\%, comparables a la reportada en la literatura. Consideramos que la combinación de 5FU en IC y vinorelbina a las dosis empleadas es un esquema efectivo en pacientes con cáncer de mama metastásico multitratadas con baja toxicidad y bajo coste.

\section{Bibliografía}

1. Breasted JH. The Edwin Smith surgical papyrus. Vol I and 22. Chicago: University of Chicago Oriental Institude Publication; 1930.

2. Compendio de cáncer $/ 2000$, mortalidad/morbilidad, Dirección General de epidemiología Secretaría de Salud, México.

3. Paul M, Hearne S, Leach D, et al. Invasive Breast cancer. En: Anderson MD. $2^{\text {nd }}$ ed. Marbán; 2000. p.13-37.

4. Acuña R, Langhi M, Machiavelli M. Vinorelbina and placlitaxel as first-line chemotherapy in metastatic breast cancer. J Clin Oncol. 1999;1:74-81.

5. Henderson IC. Chemotherapy for metastatic disease. En: Harris JR, Hellmann S, Henderson IC, et al, editors. Breast diseases. Philadelphia PA: Lipinncott; 1991. p. 604-65.

6. Bonadonna G, Giannia AM. High dose chemotherapy an autologous bone marrow transplant for adyuvant treatment of poor-risk breast cancer. Oncol J Club. 1990;2:3-6.

7. Eddy DM. High dose chemotherapy with autologous bone transplantation for the treatment of metastasic breast cancer. J Clin Oncol. 1991;10:657-70.
8. Aapro MS, Harper P, Johnson SA. Developments in cytotoxic chemotherapy: advances in treatment utilising vinorelbina. Critical reviews in Oncology/Hematology. 2001;40:251-63.

9. Leung P, Tannok L, Oza A, et al. Cost Utility analysis of chemotherapy using paclitaxel, docetaxel or vinorelvine for patients with anthracycline-resistant breast cancer. J Clin Oncol. 1999;17:3082-90.

10. González JL, Brito WO, González JF, et al. 5-Fluoruracil and vinorelbina therapy for stage IV pretreated breast cancer in Monterrey México. Pros Am Soc Clin Oncol. 2001;20:1926.

11. Garcia-Conde J, Lluch A, Martin M, et al. A phase II trial of weekly IV vinorelbina in first-line advanced breast cancer chemotherapy. Ann Oncol. 1994;5:854-7.

12. Esteba E, de Sande G, Puertas J, et al. A phase trial of cyclophosphamide, epirrubicin and vinorelbina in the treatment of advanced breast cancer. Breast Cancer Res Tret. 2000;62:127-33.

13. Gasparini G, Caffo O, Barni S, et al. Vinorelbina is an active antiproliferative agent in pretreated advanced breast cancer patients: a phase II study. J Clin Oncol. 1994; 12:2094-101.

14. Romero A, Ravinovich MG, Vallejo CT, et al. Vinorelbina as first line chemotherapy for metastatic breast cancer. $\mathrm{J}$ Clin Oncol. 1994;12:336-41.

15. Rossi A, Gridelli C, Gebbia V, et al. Single agent vinorelbina as first-line chemotherapy in elderly patients with advanced breast cancer. Anticancer Res. 2003;23(2C):1657-64.

16. Treyer G, Delozier M, Lichinister M, et al. Phase II study of oral vinorelbina in first line advanced breast cancer chemotherapy. J Clin Oncol. 2003;21:35-40.

17. Spicer DV, Ardalan B, Daniels JR, et al. Reevaluation of the maximum tolerated dose of continuous venous infusion of 5-fluoruracil with pharmacokinetics. Cancer Res. 1988;48:459-61.

18. Lokich JJ, Ahigren JD, Gullo JJ, et al. A prospective randomized comparison of continuous infusion with a conventional bolus schedule in metastatic colorectal carcinoma: a Mid-Atlantic Oncology Program Study. J Clin Oncol. 1989;7:425-32.

19. Regazzoni S, Pesce G, Cavalli G, et al. A low-dose continous infusion of 5-fluoruracil for metastatic breast cancer. Ann Oncol. 1996;7:807-13.

20. Zaniboni A, Meriggi F, Arcangeli G, et al. L-folinic acid and 5-fluoruracil in the treatment of advanced breast cancer: a phase II study. Ann Oncol. 1993;4 2 Suppl: 41S-3. 
21. Hochster HS, Vogel CL, Burman SL, et al. Activity and safety of vinorelbina combined with doxorrubicin or fluoruracil as first-line therapy in advanced breast cancer: A stratified phase II study. The Oncologist. 2001;6: 269-77.

22. Hansen R, Quebbeman E, Beatty P, et al. Continuous 5fluorouracil infusion in refractory carcinoma of the breast. Breast Cancer Res Treat. 1987;10:145-9.

23. Dieras V, Extra JM, Bellissant E, et al. Efficacy and tolerance of vinorelbina and fluorouracil combination as first-line chemotherapy of advanced breast cancer: Results of a phase II study using a sequential group method. J Clin Oncol. 1996;14:3097-104.

24. Nole F, de Braud F, Aapro M, et al. Phase I-II study of vinorelbina in combination with 5-fluorouracil and folinic acid as first-line chemotherapy in metastatic breast cancer: A regimen with a low subjective toxic burden. Ann Oncol. 1997;8:865-70.
25. Stuart N, Mc Murray M, Price C, et al. Phase II study of vinorelbina and continuous infusion 5.fluoruracil in metastatic breast cancer following anthracyclines. Proc Am Soc Clin Oncol. 2001. (Abstract 1923).

26. Berruti A, Sperone P, Bottini A, et al. Phase II Study of Vinorelbina With Protracted Fluorouracil Infusion as a Second- or Third-Line Approach for Advanced Breast Cancer Patients Previously Treated With Anthracyclines. J Clin Oncol. 2000;18:3370-7.

27. Chung CT, Carlson W. Goal and objectives in the management of metastatic breast cancer. The Oncologist. 2003;8:514-20.

28. Weber BL, Vogel C, Jones S, et al. Intravenous vinorelbina as first-line and second-line therapy in advanced breast cancer. J Clin Oncol. 1995;13:2722-30. 